\title{
Cuidado especializado a neonato prematuro fundamentado en la teoría general del autocuidado
}

\section{Premature neonate specialized care based on the general theory of self-care}

\section{Cuidado especializado a neonato prematuro fundamentado na teoria general do autocuidado}

\author{
F. Sánchez-Quiroz ${ }^{\text {a* }}$ L. Álvarez-Gallardo ${ }^{\mathrm{b}}$ \\ ${ }^{a}$ División de Estudios de Posgrado de Enfermería, Instituto Nacional de Pediatría, Escuela Nacional de enferme- \\ ría y Obstetricia, Universidad Nacional Autónoma de México; Ciudad de México, México

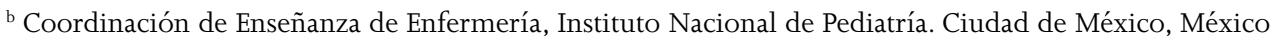

Recibido: 17 junio 2017

Aceptado: 10 agosto 2018

\section{Resumen}

Introducción: Los nacimientos prematuros permanecen como un problema perinatal, afectan entre 5 y $12 \%$ de los nacimientos, la principal condición clínica asociada a estos es el Síndrome de Dificultad Respiratoria (SDR), inicia inmediatamente al nacimiento con dificultad respiratoria progresiva que requiere asistencia ventilatoria e ingreso a Unidades de Cuidados Intensivos Neonatales (UCIN). Se presenta el abordaje de manera integral a una recién nacida de 32 SDG con SDR, en un hospital de segundo nivel de atención, en este se aplicó el proceso enfermero.

Objetivo: Brindar atención integral e individualizada con cuidados específicos al neonato prematuro, con base en la Teoría General del Autocuidado.

Método: Selección de paciente a conveniencia en UCIN, aplicación de la metodología del proceso enfermero, valoración basada en los requisitos universales de autocuidado. La información obtenida fue a través del expediente clínico, entrevista a la madre y valoración de la recién nacida. Se analizaron los datos, se emitieron diagnósticos enfermeros, se ejecutó plan de cuidados fundamentados por artículos científicos y guías de práctica clínica.

Resultados: Se realizaron 12 diagnósticos de enfermería, 10 reales y 2 de riesgo de acuerdo con los requisitos de autocuidado universal, se identificaron los más alterados: mantenimiento de un aporte suficiente de aire, agua y alimentos, y provisión de cuidados asociados con los procesos de excreción.

Conclusiones: Se lograron intervenciones independientes e interdependientes; sin embargo, el neonato permaneció en UCIN debido a que su atención es compleja y requiere personal capacitado con 
conocimientos y habilidades fundamentadas para el cuidado. La asistencia al prematuro debe ser inmediata para la detección precoz y manejo de patologías frecuentes.

Palabras clave: Síndrome de dificultad respiratoria del recién nacido; prematuro; atención de enfermería; México.

\section{Abstract}

Introduction: Premature births remain a perinatal problem affecting between $5 \%$ and $12 \%$ of cases. The main clinical condition associated is the Neonatal Respiratory Distress Syndrome (NRDS), which onsets right after birth, and which is characterized by a progressive respiratory difficulty which requires ventilation assistance at the Neonatal Intensive Care Units (NICU). This study presents the integral handling of the case of a newly born with 32 gestational weeks and NRDS at a second level of attention hospital. The Nursing Process was applied.

Objective: To provide integral and individualized attention with specific care to this premature neonate, based on the General Theory of Self-Care.

Method:The patient was selected by convenience from the NICU applying the methodology of the nursing process, and with an assessment based on the universal requirements of self-care. The related information was obtained through the clinical record, an interview to the mother, and the assessment of the patient. Data were analyzed and nursing diagnoses were generated. The care plan was executed with the support of scientific articles and clinical practice guides.

Results: 12 nursing diagnoses were emitted, 10 real and 2 of risk, including: maintenance of enough air, water, and feeding supply, and excretion related associated care.

Conclusions: Independent and inter-dependent interventions were achieved, however, the premature neonate remained at the NICU because of the complexity of the needed care, which requires constant supervision from experienced and skillful personnel. The attention to these cases must be immediate in order to handle the frequent associated pathologies.

Keywords: Respiratory distress syndrome, newborn; premature; nursing care; Mexico.

\section{Resumo}

Introdução: Os nascimentos prematuros permanecem como um problema perinatal, afeitam entre 5 e $12 \%$ dos nascimentos, a principal condição clínica associada a estresse é a Síndrome de Dificuldade Respiratória (SDR), inicia imediatamente no nascimento com dificuldade respiratória progressiva que requer assistência ventilatória e ingresso a Unidades de Cuidados Intensivos Neonatal (UCIN). Apresenta-se a abordagem de maneira integral a uma recém-nascida de 32 SDG com SDR, em um hospital de segundo nível de atenção, neste aplicou-se o processo enfermeiro.

Objetivo: Proporcionar atenção integral e individualizada com cuidados específicos no neonato prematuro, com base na Teoria General do Autocuidado.

Método: Seleção de paciente a conveniência em UCIN, aplicação da metodologia do processo enfermeiro, valoração baseada nos requerimentos universais de autocuidado. A informação obtida foi a través do expediente clínico, entrevista à mãe e valoração da recém-nascida. Analisaram-se os dados, emitiram-se diagnósticos enfermeiros, executou-se o plano de cuidados fundamentados por artigos científicos e guias de prática clínica.

Resultados: Realizaram-se 12 diagnósticos de enfermagem, 10 reais e 2 de risco conforme com os requerimentos de autocuidado universal, identificaram-se os mais alterados: mantimento de um aporte suficiente de ar, água e alimentos, e provisão de cuidados associados com os processos de excreção.

Conclusões: Conseguiram-se intervenções independentes e interdependentes; porém, o neonato permaneceu em UCIN devido a que sua atenção é complexa e requer pessoal capacitado com conhecimentos e habilidades fundamentadas para o cuidado. A assistência no prematuro deve ser imediata para a deteç̧ão precoce e manejo de patologias frequentes. 
Palavras chave: Síndrome do desconforto respiratório do recém-nascido; prematuro; cuidados de enfermagem; México.

\section{Introducción}

La primera etapa de la vida que marca el futuro del ser humano es sin duda el nacimiento, para llegar a este sucede un proceso, es decir una etapa perinatal que será determinante, de esta dependerá si es un nacimiento prematuro o a término. Los principales factores de riesgo para un nacimiento prematuro son aquellos derivados del estado de salud materno, sin embargo, no se descartan aquellos del desarrollo fetal. Dentro de los principales se encuentran: peso extremo de la madre ya sea bajo peso u obesidad, edad materna extrema $(<16$ o $>35)$, tabaquismo, situaciones de estrés, infecciones del tracto genital, rotura prematura de membranas, hemorragia anteparto, insuficiencia cervical y anormalidades uterinas congénitas ${ }^{1}$.

La Organización Mundial de la Salud (OMS), considera como prematuros a aquellos nacidos vivos antes de que se hayan cumplido 37 semanas de gestación ${ }^{2}$, son nacimientos de alto riesgo que requieren de una atención especializada. De acuerdo con este mismo Organismo en México, se estima una tasa de 7.3 nacimientos prematuros por cada 100 nacimientos. Asimismo, cabe resaltar que actualmente esta condición continúa siendo un problema perinatal importante, ya que afecta entre 5 y $12 \%$ de los nacimientos ${ }^{3}$.

La principal condición clínica asociada a la prematurez es el Síndrome de Dificultad Respiratoria (SDR), que inicia de manera inmediata al nacimiento con dificultad respiratoria progresiva, que requiere asistencia ventilatoria e ingreso a la Unidad de Cuidados Intensivos Neonatales (UCIN).

Con el objetivo de brindar atención integral e individualizada a través de cuidados específicos al neonato prematuro, se realizó el presente estudio de caso a una recién nacida con los diagnósticos médicos de: prematurez de 32 semanas de gestación (SDG) más Síndrome de Dificultad Respiratoria, hospitalizada en el servicio de UCIN de un Hospital de Segundo Nivel de atención. A su vez, se implementó el método enfermero en sus cinco etapas, así como la aplicación de la Teoría General del Autocuidado, ya que en esta se plantea que el eje fundamental de la enfermería es identificar el déficit del autocuidado y la demanda terapéutica de cuidado de los pacientes siempre y cuando cubran los requisitos universales, a fin de limitar las desviaciones de la salud para proporcionar una atención de calidad ${ }^{4}$, además de realizar intervenciones de enfermería que contribuyan en la mejora del tratamiento y de la calidad de vida de la paciente.

Por tanto, este trabajo integró una base científica para su aplicación en la práctica profesional, ya que se trata de una recién nacida que requiere de la total atención de enfermería y de cuidados especializados, dado que el Síndrome de Dificultad Respiratoria es la complicación más frecuente de la prematurez, y más de la mitad de los recién nacidos con un peso al nacer de entre 501 y 1500 gramos muestran signos de esta patología. El principal tratamiento en estos neonatos, es la aplicación del factor surfactante, diversos estudios demuestran que este reduce la mortalidad y cualquier forma de pérdida de aire pulmonar en alrededor de un 30\% y un 50\%, respectivamente; de este modo, la administración de este es primordial, en tanto se ha demostrado que los neonatos que reciben surfactante profiláctico (administrado inmediatamente después del parto, antes de que el neonato desarrolle dificultad respiratoria) presentan mejores resultados que aquellos que reciben surfactante de rescate (administrado solamente a los que desarrollan dificultad respiratoria $)^{5}$.

De igual modo, las complicaciones o patologías relacionadas con la prematuridad continúan siendo la principal causa de defunción en los niños menores de 5 años, que en el año 2013 provocaron cerca de un millón de muertes y tres cuartas partes de esas muertes podrían evitarse con intervenciones actuales y eficaces ${ }^{6}$, también muchos de los neonatos prematuros que sobreviven sufren algún tipo de discapacidad de por vida; en ello radica la importancia de que el personal de enfermería esté preparado con conocimientos científicos y especializados, para realizar seguimiento en la prescripción del cuidado. 


\section{Métodos}

La realización de este estudio de caso se llevó a cabo en un Hospital de Segundo Nivel de atención, se seleccionó a la persona recién nacida a conveniencia en la unidad de cuidados intensivos neonatales y se implementó la metodología del proceso enfermero; durante la etapa de valoración se utilizó una Guía basada en la Teoría General del Autocuidado con aplicación del método clínico; la recolección de datos se realizó por medio del expediente clínico, entrevista al cuidador primario y valoración integral de la recién nacida con previa autorización de la madre quien firmó el consentimiento informado. Posteriormente, se analizaron los datos obtenidos y se emitieron diagnósticos de enfermería; para su desarrollo se puso en práctica el pensamiento crítico.

Así, al continuar con el estudio, se realizaron intervenciones especializadas que se fundamentaron con los procedimientos de la Enfermería Basada en Evidencia, se realizó una revisión bibliográfica de artículos científicos de distintos países incluido México, así como guías de práctica clínica.

Finalmente, durante la evaluación se logró identificar el cumplimiento de los objetivos planteados en cada uno de los diagnósticos emitidos, sin embargo la persona recién nacida permaneció en UCIN con un estado de salud grave y por tanto no fue posible realizar un plan de alta de enfermería, pero se llevó un control del caso.

\section{Resultados}

\section{Valoración}

\section{Factores básicos condicionantes:}

Paciente neonato, será identificada como BC, que es ingresada al servicio de UCIN; dentro de los factores del sistema familiar BC cuenta con una familia extensa de ocho integrantes, que habitan en el mismo domicilio y tienen una relación afectiva fuerte; en cuanto a los factores del sistema sociocultural se encuentra que sus padres, ambos adolescentes, madre de 15 años que se dedica al hogar con estudios a nivel de secundaria terminada, padre de 17 años que es empleado de una fábrica con estudios igualmente a nivel secundaria; BC no fue planeada pero es aceptada por la familia, sus padres conviven en unión libre, habitan en una zona urbana, en casa de sus abuelos y cuentan con todos los servicios intradomiciliarios, sin fauna nociva; respecto a servicios de salud, están afiliados al seguro popular de la Secretaria de Salud.

Requisitos de autocuidado en el desarrollo:

$\mathrm{BC}$ al nacimiento obtuvo una calificación Apgar de 7/9, lloró y respiró al nacer, a los 10 minutos de vida requirió intubación endotraqueal para mejorar el patrón respiratorio, así como de la administración de una dosis de surfactante exógeno. Por otra parte, al momento de la valoración de la edad gestacional por el método Capurro se obtuvieron 32 semanas de gestación (Gráfica 1) y de acuerdo a la gráfica del Dr. Jurado García, se encontró en un peso adecuado para la edad gestacional dentro del percentil 10.

Requisitos de desviación a la salud:

Madre primigesta, sin antecedentes de transfusiones previas ni intervenciones quirúrgicas, negó alergias. Única hospitalización durante el parto actual, asistió a nueve consultas para control prenatal a partir del 1 er. mes de embarazo en un centro de salud y un ultrasonido sin anormalidades en la 21 SDG. Aparentemente cursó un embarazo normoevolutivo, sin patologías durante el mismo. Presentó ruptura prematura de membranas a las 32 SDG e inmediatamente acudió a facultativo, debido a la condición de parto pretérmino se le aplicaron dos dosis de Betametasona para maduración pulmonar, evolucionó a 89 horas de ruptura de membranas, la cesárea fue programada y se llevó a cabo con anestesia por bloqueo epidural para intervenir y se obtuvo un producto único vivo. 


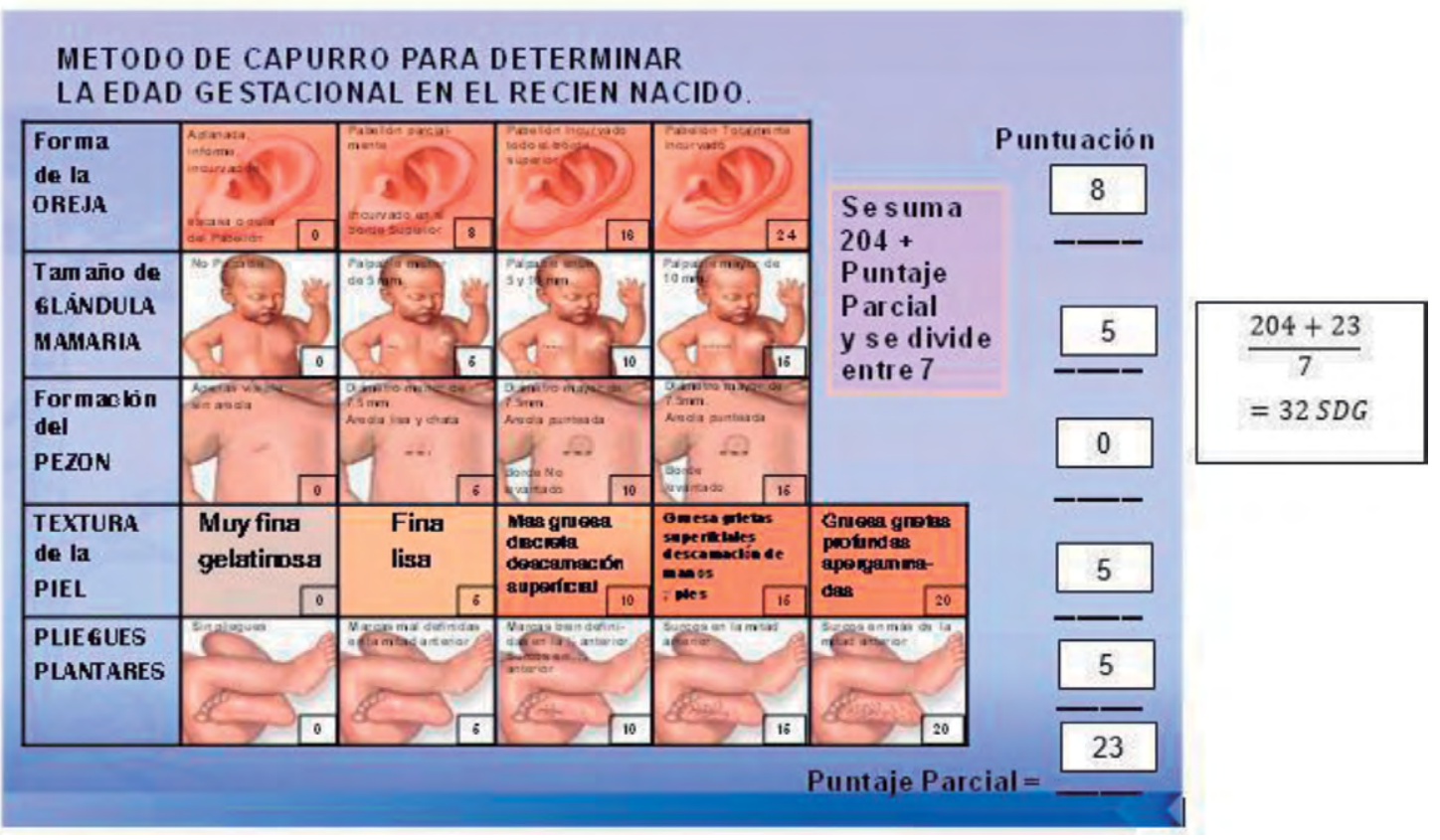

Gráfica I. Método Capurro. ${ }^{7}$

\section{Habitus exterior}

Neonato prematuro femenina, en cuna de calor radiante cursa su tercer día de vida extrauterina, aparentemente con peso adecuado para edad gestacional, palidez generalizada de tegumentos con monitorización de signos vitales y pulsioximetría, continua manteniendo $\mathrm{SpO} 2>90 \%$; en Fase III de ventilación, con respuesta a estímulos externos, irritabilidad y facies de dolor al manejo, con apoyo hidroelectrolítico vía parenteral, cuna en posición semi fowler y vestimenta sólo con pañal grande a su edad.

\section{Requisitos de autocuidado universal.}

Mantenimiento de un aporte suficiente de aire: nariz simétrica en línea media, pequeña, fosas nasales permeables sin presencia de secreciones y mucosa hidratada. Cuello simétrico, integro sin adenomegalias con movimientos de flexión y extensión presentes pero limitados por presencia de cánula orotraqueal, tráquea en línea media correspondiente, a la palpación presencia de discos íntegros, sin alteraciones, hombros simétricos, con clavículas integras. Tórax normolíneo, simétrico, adecuada implantación de mamas, costillas adecuadamente implantadas, ritmo respiratorio apoyado por ventilación mecánica con disminución en la profundidad de tórax durante la inspiración y espiración, así como disminución de la expansión torácica bilateral espontánea. Presentó datos de dificultad respiratoria con una valoración de Silverman-Anderson de 3 puntos por tiraje intercostal acentuado y quejido respiratorio leve, una frecuencia respiratoria de $61 \mathrm{rpm}$, a la auscultación campos pulmonares ventilados con estertores gruesos bilaterales y aspiración de abundantes secreciones hialinas por cánula y orofarínge. Se encuentra en Fase III de la ventilación en modalidad BIPAP en parámetros establecidos PIP 20, PEEP 4, Fr 65, T Insp 0.36, FiO2 100\%. Es portadora de sonda pleural 2 Fr con sello de agua a succión continua por neumotórax izquierdo, se ausculta burbujeo e hipoventilación; sin embargo, no se logra observar radiografía de tórax. Ruidos cardiacos rítmicos sin presencia de soplos con una frecuencia cardiaca de 145 $\mathrm{x}^{\prime}$, con presencia de pulsos distales rítmicos de buena intensidad y una tensión arterial de 80/57 (68). Mantiene saturación de oxigeno $>90 \%$ y llenado capilar de 2". Mantenimiento de un aporte suficiente de líquidos y alimentos: cráneo normocéfalo con adecuada implantación de cabello color café oscuro, fontanela bregmática normotensa, fontanela lamboidea puntiforme; suturas bien afrontadas palpables con coronarias ligeramente separadas, perímetro cefálico de $28 \mathrm{~cm}$. Piel íntegra, fina y delgada con 
adecuada hidratación, boca íntegra, simétrica con presencia de ambos labios rosados, paladar integro, lengua pequeña rosada, frenillos íntegros. Ausencia del reflejo de búsqueda, succión débil y deglución ausente hasta el momento, con cánula orotraqueal y sonda orogástrica. Abdomen distendido que luce lustroso con perímetro abdominal de $24.5 \mathrm{~cm}$ con leve dibujo de asas intestinales y peristalsis ausente, por lo que se mantiene en ayuno con sonda orogástrica a derivación con contenido gástrico de $20 \mathrm{ml}$ $(>100 \%)$. Muñón umbilical en proceso de momificación sin datos de infección y sin visceromegalias palpables sin agregados. Aporte calórico por vía parenteral con NPT $64.8 \mathrm{Kcal} / \mathrm{kg} /$ día, con líquidos totales de 130-150 ml/kg/día a través de PICC 2 Fr en MSD, además de contar con Catéter Periférico 24 Fr en MID funcional. En relación con el peso tuvo una ganancia de 20 gr. en 3 días, sin incremento de la talla, con glicemia capilar de 71-87 mg/dl.

Procesos de eliminación y excretas: genitales íntegros de acuerdo a edad y fenotípicamente de sexo femenino con labios mayores prominentes sin llegar a cubrir labios menores, sin lesiones aparentes. Presenta eliminación urinaria espontánea con un índice urinario de $3.2 \mathrm{ml} / \mathrm{hr}$, uresis amarilla sin olor fétido, no es posible medir densidad urinaria. En cuanto a eliminación fecal no presenta evacuaciones, con presencia de asas intestinales y abdomen distendido. Sus pérdidas insensibles calculadas fueron de $20.3 \mathrm{ml}$ por turno con $2.9 \mathrm{ml}$ por hora.

Mantenimiento del equilibrio entre la actividad y reposo: ojos simétricos con escasa implantación de cejas y pestañas en parpados, reflejo pupilar y fotomotor muy disminuido, pupilas isocóricas, esclerótica con hematoma alrededor de pupila con mínima ruptura de capilares que se asocia a trauma obstétrico y reflejo glabelar aún no desarrollado. Oídos íntegros con orejas pequeñas con curvatura delgada y pabellón auricular adecuadamente implantado, sin lesiones aparentes. Columna vertebral íntegra, no es valorable la simetría debido a disminución en la movilidad por apoyo mecánico ventilatorio, no obstante son palpables cervicales. Cadera simétrica e integra, Barlow y Ortolani negativo, con adecuado movimiento de flexión y extensión en coordinación con extremidades inferiores. Extremidades íntegras simétricas con longitud de acuerdo a talla, ortejos bien implantados sin aumento en cantidad, pliegues palmares y plantares tenues por edad, con adecuado tono muscular. En cuanto a los reflejos primarios se encuentran ausentes, reflejo de moro ausente y tónico de cuello ausente; reflejo de enderezamiento e inervación del tronco no valorables, ya que no es factible la movilización por presencia de sello de agua y ventilación mecánica, reflejo de puntos cardinales ausente, presión palmar y plantar ausentes, adecuado tono muscular y Babinsky positivo. De acuerdo a la escala Brazelton el neonato se encontró en un estado de conciencia I, con sueño profundo, respiración regular con asistencia mecánica ventilatoria, ojos cerrados la mayor parte del tiempo, sin actividad espontánea a excepción de los sobresaltos e irritabilidad por el manejo, esto se asocia a la prematurez del neonato y agravamiento de su estado de salud.

En la valoración con la teoría sinactiva la recién nacida se mostró en el sistema autonómico con signos de estrés, ya que se encuentra en Fase III de la ventilación, por lo que durante la aspiración de secreciones muestra irritabilidad y en el sistema motor se encontró con signos de estabilidad durante el curso de sueño, ya que a la manipulación se alteraba este sistema, con presentación de flexión de extremidades (desorganización corporal).

Mantenimiento del equilibrio entre la soledad e interacción social: se trata de neonato no planeada, hija de madre y padre adolescentes; durante la visita ambos padres establecen poco contacto físico con ella, se observa miedo por la hospitalización.

Prevención de los peligros para la vida y el bienestar humano: debido a la hospitalización el neonato se encuentra expuesto a los peligros medio ambientales, como el ruido, afección de la iluminación, que incluye riesgo de infección por procedimientos invasivos y riesgo de caídas por la edad. La madre de la recién nacida es primigesta en etapa de desarrollo de la adolescencia, sin conocimientos sobre los cuidados que se deben brindar a la recién nacida durante y fuera de la hospitalización, y en este caso con mayor complejidad por la condición de prematurez. 
Se valoró el riesgo de ulceras por presión alto con 11 puntos (NSRAS) y en la valoración del dolor se encontró mínimo con 4 pts. de la escala PIPP con facies de dolor, además cuenta con infusión de Fentanyl como analgesia continua a $0.2 \mathrm{~mL} / \mathrm{h}(4 \mathrm{mcg} / \mathrm{Kg} / \mathrm{h})$.

Promoción de la normalidad: la adaptación del neonato a la vida extrauterina no es favorable debido a la prematurez, ya que durante la valoración se encontró dificultad en el patrón respiratorio con necesidad de apoyo mecánico en la ventilación. Se encuentra con respuesta a estímulos externos con irritabilidad al manejo; adecuado tono muscular en extremidades, reflejos primitivos ausentes e incapacidad para iniciar una alimentación trófica, por tanto se mantiene con aporte calórico vía parenteral.

\section{Diagnóstico}

Una vez realizada la valoración de requisitos de autocuidado universal de acuerdo con la Teoría del Déficit de Autocuidado de Dorothea Orem, se realizaron 12 diagnósticos de enfermería con formato PES (problema, etiología, signos y síntomas), 10 reales y 2 de riesgo, de acuerdo al estado de salud de la paciente y a la aplicación del proceso enfermero:

1. Incapacidad para eliminar secreciones de la vía aérea $\mathrm{r} / \mathrm{c}$ retención de las secreciones $\mathrm{m} / \mathrm{p}$ estertores gruesos bilaterales y abundantes secreciones por cánula orotraqueal y orofarínge.

2. Déficit de la función respiratoria $\mathrm{r} / \mathrm{c}$ aumento en la tensión a nivel alveolar $\mathrm{m} / \mathrm{p}$ hipoventilación de base pulmonar izquierda por neumotórax y Silverman de 3 puntos por presencia de tiraje intercostal acentuado y quejido respiratorio leve.

3. Incapacidad para absorber nutrientes $\mathrm{r} / \mathrm{c}$ prematuridad $\mathrm{m} / \mathrm{p}$ distensión abdominal, dibujo de asas intestinales y residuo gástrico $>100 \%(20 \mathrm{ml})$.

4. Incapacidad para iniciar la alimentación enteral $\mathrm{r} / \mathrm{c}$ prematuridad $\mathrm{m} / \mathrm{p}$ succión ausente y alteración de la función respiratoria.

5. Riesgo de deterioro en la mucosa oral $\mathrm{r} / \mathrm{c}$ instalación de cánula orotraqueal y sonda orogástrica.

6. Estreñimiento $\mathrm{r} / \mathrm{c}$ motilidad gastrointestinal ausente $\mathrm{m} / \mathrm{p}$ distención abdominal, peristalsis ausente e incapacidad para eliminar heces.

7. Bienestar del proceso de eliminación urinaria $\mathrm{m} / \mathrm{p}$ índice urinario de $3.2 \mathrm{ml} / \mathrm{Kg} / \mathrm{h}$ dentro de parámetros normales.

8. Alteración del estado de sueño $\mathrm{r} / \mathrm{c}$ factores ambientales hospitalarios $\mathrm{m} / \mathrm{p}$ desorganización corporal con flexión de extremidades en $\mathrm{W}$ e irritabilidad al manejo.

9. Desequilibrio del vínculo madre-hijo $\mathrm{r} / \mathrm{c}$ hospitalización del neonato $\mathrm{m} / \mathrm{p}$ ausencia de contacto.

10. Dolor $\mathrm{r} / \mathrm{c}$ procedimientos y manipulación excesiva $\mathrm{m} / \mathrm{p}$ facies de dolor y escala PIPP de 4 puntos.

11. Riesgo de infección $\mathrm{r} / \mathrm{c}$ dispositivos invasivos instalados (Catéter PICC, ventilación mecánica).

12. Desequilibrio del bienestar humano r/c déficit de conocimientos de los padres sobre el cuidado de recién nacido $\mathrm{m} / \mathrm{p}$ miedo de los padres para tener contacto con la recién nacida.

\section{Planeación}

De acuerdo a los sistemas de enfermería de la teoría de Dorothea Orem se activó el sistema de enfermería totalmente compensatorio, la agencia de enfermería es quien implementó totalmente el plan de cuidados, ya que el estado clínico de la paciente no permitió en este caso, que la madre como agente de cuidado dependiente participara de manera total en los cuidados.

Cabe destacar que al desarrollar el plan de cuidados se utilizaron indicadores para valorar la eficacia de este, que fueron establecidos de acuerdo a los diagnósticos, sin embargo para estos no se utilizó escala Likert o Diana, pero se aplicaron como indicadores de bienestar.

Posteriormente, una vez realizados los diagnósticos de enfermería para poder realizar la ejecución de las intervenciones, se tomaron en cuenta los riesgos sobre los que se encontraba expuesta la paciente en cuanto a persona y ambiente, tales como riesgo de sepsis, caídas, deterioro neurológico y deterioro del rol parental, principalmente. 


\section{Ejecución}

El plan de cuidados se organizó de acuerdo a los diagnósticos de enfermería identificados, se jerarquizaron los problemas encontrados. Se desarrollaron cinco diagnósticos de los 12 identificados; las intervenciones se realizaron con base en la taxonomía NIC de intervenciones en enfermería, adaptadas a la condición clínica del neonato.

Diagnóstico 1. Requisito 1: Mantenimiento de un aporte suficiente de aire.

Incapacidad para eliminar secreciones de la vía aérea $\mathrm{r} / \mathrm{c}$ retención de secreciones $\mathrm{m} / \mathrm{p}$ estertores gruesos bilaterales y abundantes secreciones por cánula orotraqueal y orofarínge.

Objetivo: mantener una vía aérea permeable en la paciente durante el tiempo que permanezca en Fase III de ventilación, de esta forma evitar una ventilación inefectiva.

Indicadores: estertores, frecuencia respiratoria, presencia de secreciones.

Intervención de enfermería: Aspiración de secreciones.

Acciones:

- Colocar el oxímetro de pulso y evaluar los niveles de SpO2.

- Mantener en posición semifowler para favorecer la respiración.

- Auscultar campos pulmonares y evaluar la presencia de sonidos agregados.

- Determinar la necesidad de aspiración orotraqueal y por orofarínge.

- Realizar la aspiración de secreciones, además de monitorizar la oxigenación y los parámetros ventilatorios.

Intervención de enfermería: Fisioterapia pulmonar.

Acciones:

- Mantener la cuna del neonato en posición semi fowler y el drenaje postural para favorecer la movilización de las secreciones.

- Apoyar con digitovibración en tórax para eliminar las secreciones, si no es posible la movilización del neonato.

- Valorar la mecánica de la respiración durante la fisioterapia.

- Auscultar campos pulmonares para determinar el lugar de acumulación de secreciones.

- Evaluar si la fisioterapia fue efectiva posterior a la aspiración de secreciones.

Fundamentación de las intervenciones:

Mantener una vía aérea permeable favorece la ventilación de todos los segmentos pulmonares y mantienen un drenaje de las secreciones. A su vez, permite la relajación del musculo liso pulmonar con lo cual se disminuye el esfuerzo respiratorio y el broncoespasmo ${ }^{8}$. El tratamiento de la fisioterapia pulmonar está basado fundamentalmente en técnicas de estimulación y relajación. Tenemos que tener en cuenta siempre las siguientes premisas:

- Extremada fragilidad de árbol bronquial.

- Semana de gestación y peso del RN.

- Contexto de la ventilación mecánica, con respeto de los tiempos de ventilación impuestos por el niño o por el respirador.

- Vigilancia y técnica adecuada en la aspiración?

Evaluación: paciente que se mantiene en Fase III de ventilación, con disminución de los parámetros de FiO2 hasta $60 \%$ y ciclado de $25 \mathrm{rpm}$, lo cual indica que hay una mejora en el patrón respiratorio, continua con signos vitales en parámetros normales y adecuada oxigenación evaluada a través de oxímetría de pulso, también mediante la aspiración de secreciones y la fisioterapia pulmonar proporcionada con 
digitovibración en tórax, se eliminaron las secreciones orofaríngeas lo que contribuyó a mejorar el patrón respiratorio y la saturación de oxígeno.

Diagnóstico 2. Requisito 1: Mantenimiento de un aporte suficiente de aire

Déficit de la función respiratoria $\mathrm{r} / \mathrm{c}$ aumento en la tensión a nivel alveolar $\mathrm{m} / \mathrm{p}$ hipoventilación de base pulmonar izquierda por neumotórax y Silverman de 3 puntos por presencia de tiraje intercostal acentuado y quejido respiratorio leve.

Objetivo: mejorar la oxigenación con mantenimiento de parámetros normales de $\mathrm{SpO}_{2}$ para evitar la hipoxia y deterioro pulmonar, durante el tiempo que requiera asistencia respiratoria.

Indicadores: entrada y salida del aire pulmonar, datos de dificultad respiratoria.

Intervención de enfermería: Valoración de la función respiratoria.

Acciones:

- Auscultar periódicamente el tórax para determinar la presencia de sonidos respiratorios adventicios.

- Evaluar la escala Silverman Anderson y determinar el nivel de dificultad respiratoria.

- Mantener la cabecera de la cuna en posición semi-fowler.

- Evaluar el nivel de oxigenación a través de la oximetría de pulso continua.

- Valorar las causas de hipoventilación pulmonar y tratar la causa con apoyo del tratamiento médico.

Intervención de enfermería: Cuidados del drenaje pleural.

Acciones:

- Determinar la adecuada colocación de la sonda pleural en la paciente.

- Comprobar la funcionalidad de la cámara de control de aspiración, para una aspiración correcta en el drenaje pleural.

- Revisar el nivel de líquido en la cámara de agua sellada; mantener a nivel menor a 12 cmH2O.

- Evitar una presión de aire excesiva que agrave el problema.

- Observar burbujas en la cámara de agua sellada de lo contrario comprobar succión y nivel de agua.

- Verificar que no haya escape de aire y que la sonda pleural se encuentre fija; evaluar características del drenado.

Fundamentación de las intervenciones:

Se debe mantener el intercambio gaseoso hasta que el esfuerzo respiratorio del recién nacido sea adecuado, con base en los índices de ventilación pulmonar, así como las interacciones entre ellos y los cambios que producen en la fisiología pulmonar, por ello una adecuada valoración de la función pulmonar será necesaria ${ }^{10}$.

La colocación de un tubo de drenaje pleural es el tratamiento de elección para el neumotórax, debiéndose llevar un adecuado control en el nivel de succión y sello de agua. También, es necesario evaluar la funcionalidad respiratoria para determinar la posibilidad de retirar el drenaje lo más pronto posible, ya que los objetivos del drenaje pleural son la evacuación de aire, la re insuflación del pulmón y la prevención de recaídas en cuanto a neumotórax ${ }^{11}$.

Evaluación: se colabora en el retiro de la sonda pleural por remisión de neumotórax, sin embargo, a la auscultación continúa con hipoventilación en base pulmonar izquierda y estertores bilaterales.

Diagnóstico 4. Requisito 2 y 3: Mantenimiento de un aporte suficiente de agua y alimentos. Incapacidad para absorber nutrientes $\mathrm{r} / \mathrm{c}$ prematuridad $\mathrm{m} / \mathrm{p}$ distensión abdominal, dibujo de asas intestinales y residuo gástrico $>100 \%(20 \mathrm{ml})$.

Objetivo: evitar la desnutrición por periodo prolongado de ayuno, a través de un adecuado aporte calórico que limite el déficit durante el tiempo que se mantenga el ayuno.

Indicadores: ausencia de alimentación enteral, ayuno.

Intervención de enfermería: Administración de la nutrición parenteral total. 
Acciones:

- Vigilar el aporte calórico necesario por día del neonato.

- Comprobar que el neonato tenga un catéter central instalado correctamente.

- Mantener una técnica estéril al preparar y colocar la NPT.

- Utilizar una bomba de infusión para el aporte por NPT.

- Peso diario para evaluar si hay ganancia de peso por NPT.

Fundamentación de las intervenciones:

El aporte de líquidos en el prematuro con SDR debe ser el suficiente para satisfacer las necesidades fisiológicas, evitar la deshidratación y pérdidas de peso mayores al 10\%. Esto reduce los riesgos de:

- Persistencia de conducto arterioso y la repercusión hemodinámica

- Enterocolitis necrosante

- Displasia broncopulmonar

- Hemorragia intraventricular

- Muerte $^{12}$

En los recién nacidos prematuros, los períodos prolongados con deficiente aporte nutricional ocasionan trastornos metabólicos graves, por lo que se debe iniciar Nutrición Parenteral Total (NPT) cuando las condiciones hemodinámicas y metabólicas estén bajo control, preferentemente dentro de las primeras 24 a 48 horas de vida ${ }^{13}$.

Evaluación: en cuanto al estado nutricional la persona recién nacida se mantiene en ayuno con un aporte calórico de $64.5 \mathrm{Kcal} / \mathrm{kg} /$ día por medio de NPT, con una ganancia de peso de 20 gr. en tres días (1305 a 1325gr), con sonda orogástrica a derivación para drenar contenido gástrico con vigilancia constante de sus características y evaluar si disminuye el vaciado gástrico.

Diagnóstico 6. Requisito 4: Provisión de cuidados asociados a los procesos de eliminación y excreción. Estreñimiento $\mathrm{r} / \mathrm{c}$ motilidad gastrointestinal ausente $\mathrm{m} / \mathrm{p}$ distención abdominal, peristalsis ausente e incapacidad para eliminar heces. Objetivo: vigilar y contribuir en mejorar el patrón de eliminación de excretas durante el periodo de hospitalización.

Indicadores: patrón de eliminación, valoración abdominal.

Intervención de enfermería: Manejo del estreñimiento.

Acciones:

- Comprobar los movimientos intestinales, así como incluir frecuencia, e intensidad.

- Vigilar la existencia de peristaltismo.

- Medir perímetro abdominal al menos dos veces por turno.

- Evaluar la presencia de dolor a la palpación abdominal.

- Realizar estimulación rectal para favorecer la evacuación si es necesario.

- Realizar ejercicios con masaje abdominal suave para favorecer la eliminación fecal.

Intervención de enfermería: Cuidados del drenaje de sonda orogástrica. Acciones:

- Evaluar las características del drenaje de la sonda orogástrica.

- Determinar si hay problemas intestinales que precise tratamiento inmediato debido a las características del contenido gástrico.

- Cuantificar el gasto obtenido de la SOG a derivación.

Fundamentación de las intervenciones: 
La falta de alimentación enteral favorece la disminución de la motilidad gastrointestinal, no obstante se deben de descartar patologías intestinales previamente. Deben vigilarse las condiciones abdominales con registro del perímetro abdominal, presencia de evacuaciones, presencia de residuos gástricos, vómitos o presencia de sangre en las evacuaciones en todos los pretérmino con SDR en quienes se haya iniciado la alimentación ${ }^{14}$.

Evaluación: la eliminación persistió comprometida, ya que no se ausculta peristalsis durante el tiempo de valoración, pero se vigila énfasis en la eliminación fecal, así como características abdominales anormales.

Diagnóstico 11. Requisito 7: Prevención de los peligros para la vida y el bienestar humano.

Dolor $\mathrm{r} / \mathrm{c}$ procedimientos $\mathrm{m} / \mathrm{p}$ facies de dolor y escala PIPP de 4 puntos.

Objetivo: limitar en lo posible la manipulación y mejorar la respuesta al dolor en el neonato durante el tiempo de hospitalización.

Indicadores: escala del dolor, facies de dolor.

Intervención de enfermería: Valoración del dolor.

Acciones:

- Utilizar escalas para valorar el dolor en el neonato.

- Observar las claves no verbales del dolor.

- Determinar la necesidad de tratamiento farmacológico para el manejo del dolor.

Intervención de enfermería: Manejo del dolor.

Acciones:

- Ministrar Fentanyl en infusión continua a $0.2 \mathrm{~m} / \mathrm{h}$.

- Valorar el efecto del tratamiento farmacológico para el manejo del dolor.

- Establecer medidas de confort que minimicen la presencia de dolor.

Fundamentación de las intervenciones:

Los recién nacidos hospitalizados experimentan una gran cantidad de manipulaciones y procedimientos diarios, frecuentemente dolorosos o al menos estresantes, entre otros aspectos, por la perturbación de sus ciclos sueño-vigilia, por lo que el control del ambiente en la Unidad de Cuidados Intensivos minimiza los signos de estrés en el paciente, por lo que promueve su recuperación y permite evaluar su estado de desarrollo ${ }^{15}$, así como el manejo del dolor es fundamental para la estabilización del neonato y para mejorar su estado de sueño y descanso. La evidencia para el uso rutinario de opioides en el recién nacido bajo asistencia mecánica a la ventilación es insuficiente, por lo que deben ser usados selectivamente conforme al juicio clínico y la posible presencia de dolor. Se administrará analgesia durante la realización de procedimientos que causen dolor moderado o grave, se recomienda la administración de fentanilo a dosis de 2-3 mcg $/ \mathrm{kg} /$ dosis.

Evaluación: el control del dolor fue constante, en el cual se mantuvo en tiempo la infusión continua de analgésico opioide y con una medición, a través de escalas de valoración para mejorar la respuesta de la paciente. Se continúa la prescripción del cuidado en espera de un mejor pronóstico.

\section{Discusión}

La aplicación del método enfermero en la Unidad de Cuidados Intensivos Neonatales permite al profesional de enfermería identificar y jerarquizar los problemas potenciales que afectan el estado de salud del neonato, para realizar intervenciones que mejoren el pronóstico, de este modo el profesional de enfermería utiliza sus opiniones, conocimientos y habilidades para diagnosticar y tratar la respuesta del paciente a los problemas reales o potenciales que presentan ${ }^{16}$. 
Durante la etapa neonatal la adaptación a la vida extrauterina depende del grado de madurez anatómica y fisiológica al nacimiento, ello determina si se requiere asistencia del profesional de la salud o si cursará con alguna patología, posteriormente. El neonato prematuro es considerado como el más vulnerable de requerir hospitalización posterior al nacimiento, ya que a pesar de que se conozcan ampliamente los riesgos a los que se encuentran expuestos aún constituyen un problema perinatal grave, por tanto, también siguen en aumento los índices de mortalidad por prematurez y sus complicaciones o las patologías asociadas a esta etapa del desarrollo, se requiere contar con las herramientas necesarias para su manejo, ya que esto permitirá limitar el daño ${ }^{17}$.

Al realizar los planes de cuidados de enfermería de manera integral, se deben tomar en cuenta las necesidades generales de los recién nacidos prematuros en relación directa a la patología, al tratamiento y a su condición de prematurez. La mayoría de los neonatos prematuros requieren de asistencia respiratoria, ya que la inmadurez primordial se encuentra a nivel pulmonar por un déficit en la secreción de surfactante lo que se denomina Síndrome de Dificultad Respiratoria, dato característico de inmadurez del centro respiratorio, responsable de crisis de apnea, para la cual se requerirá apoyo ventilatorio; en esta condición el objetivo del tratamiento es garantizar una ventilación efectiva, a menudo a través de ventilación mecánica que permita lograr una oxigenación y ventilación adecuadas, así como evitar el trauma por presión, volumen y oxígeno en aquellos pacientes con falla respiratoria grave ${ }^{18,19}$.

De acuerdo a este estudio de caso, el cuidado de enfermería es esencial en la atención del recién nacido, las intervenciones que realiza este profesional se fundamentan en evidencia científica en complemento con el uso de las teorías de enfermería, que son un parteaguas para la aplicación del proceso enfermero. En este estudio de caso se abordó la Teoría General del Autocuidado de Dorothea Orem con énfasis en la subteoría del Déficit de Autocuidado, conforme a esta las personas generan su capacidad de autocuidado, pero cuando la capacidad de la persona no es suficiente para afrontar estas demandas es cuando surge un déficit ${ }^{20}$; en este caso hablamos de un neonato que no es capaz de cumplir con las demandas de autocuidado y menos durante un proceso patológico, por tanto brinda oportunidad para que la agencia de cuidado sea el profesional de enfermería, capaz de ser aplicada y evaluada en las diferentes etapas de desarrollo por las que pasa el ser humano.

Finalmente, contribuir a disminuir la incidencia de SDR a partir de la identificación precoz de los factores de riesgo asociados, son actividades que realiza el profesional de enfermería, ya que la edad gestacional juega un papel importante mayor al peso de nacimiento, puesto que demuestran su grado de inmadurez y son parte de su pronóstico durante la transición a la vida extrauterina ${ }^{21}$.

Por ello, la importancia de que a través de las intervenciones de enfermería independientes e interdependientes se limite el daño de complicaciones e impacte el cuidado a largo plazo, en la recuperación del neonato prematuro.

\section{Conclusiones}

La asistencia al prematuro debe ser inmediata para la detección precoz y manejo de patologías frecuentes, de este modo se lograron intervenciones independientes; aún así el neonato permaneció en UCIN.

En conclusión el cuidado de enfermería dentro de la UCIN constituye el pilar fundamental en la recuperación del paciente. A través de este estudio de caso se demostró que la aplicación del método enfermero se realiza como parte de la práctica diaria de enfermería, esto permite colaborar en la mejora de los tratamientos y del cuidado individualizado e integral en el neonato; ya que su atención es compleja y requiere personal capacitado con conocimientos y habilidades fundamentadas para el cuidado.

Con base en la evidencia científica disponible, el cuidado es brindado con calidad y eficacia dentro de las Unidades de Cuidado Intensivo Neonatal, al realizar intervenciones que impacten en la recuperación del paciente, como lo fue en el caso presentado, a través de estas se logró cumplir con los objetivos planteados en cada plan de cuidados de acuerdo a la condición clínica de la paciente. 


\section{Responsabilidades éticas}

Protección de personas y animales. Los autores declaran que para esta investigación no se han realizado experimentos en seres humanos ni en animales.

Confidencialidad de los datos. Los autores declaran que han seguido los protocolos de su centro de trabajo sobre la publicación de datos de pacientes.

Derecho a la privacidad y consentimiento informado. Los autores han obtenido el consentimiento informado de los pacientes y/o sujetos referidos en el artículo. Este documento obra en poder del autor de correspondencia.

Conflicto de intereses. Los autores declaran no tener ningún conflicto de intereses.

Financiamiento. Ninguno.

\section{Referencias}

1. PerkinElmer. Nacimiento Prematuro-retos y oportunidades de la predicción y la prevención. Turku; PerkinElmer; 2009.

2. Organización Mundial de la Salud. Nota descriptiva: Nacimientos prematuros. Ginebra: OMS; 2016. https://bit.ly/2voykug

3. Pérez-Zamudio R, López-Terrones CR, Rodríguez-Barboza A. Morbilidad y mortalidad del recién nacido prematuro en el Hospital General de Irapuato. Bol. Med. Hosp. Infant. Mex. 2013; 70(4): 299-303.

4. Prado-Solar LA, González-Reguera M, Paz-Gómez N, et al. La teoría Déficit de autocuidado: Dorothea Orem punto de partida para calidad en la atención. Rev. Med. Electron. 2014; 36(6): 835-45.

5. Ballesteros-del Olmo JC, Udaeta-Mora E, Villegas-Silva R, et al. Guía de práctica clínica: Tratamiento del síndrome de dificultad respiratoria neonatal. Rev. mex. pediatr. 2011; 78(Supl.1); S3-S25.

6. Organización Mundial de la Salud. Salud neonatal: proyecto de plan de acción. Todos los recién nacidos: un plan de acción para poner fin a la mortalidad prevenible. Ginebra: OMS: 2014.

7. Gómez-Gómez M, Danglot-Banck C, Aceves-Gómez M. Clasificación de los recién nacidos. Rev. mex. pediatr. 2012; 79(1): 32-9.

8. Ministerio de Salud de Chile. Manual de procedimientos para la atención del recién nacido en el período inmediato y puerperio en servicios de obstetricia y ginecología. Santiago de Chile: Ministerio de Salud de Chile; 2013.

9. Torés E, De Frutos R, Sánchez MJ. Fisioterapia respiratoria en neonatos. Madrid: XV Jornadas Sociedad Española de Rehabilitación Infantil, 2010.

10. Villanueva-García D, Ávila-Reyes R, Dies-Suárez P, et al. PAC Neonatología-4/Libro 2/Insuficiencia respiratoria neonatal. Revisión revisada y actualizada. México: Intersistemas; 2016.

11. Martinón-Torres F, Martinón-Sánchez JM. Toracocentesis y drenaje pleural. An. pediatr. contin. 2013; 1(3): 159-65. https://doi.org/10.1016/S1696-2818(03)71608-4

12. Secretaría de Salud México. Guía de Práctica Clínica. Manejo de líquidos y electrólitos en el recién nacido prematuro en la Unidad de Cuidados Intensivos Neonatales. Ciudad de México: CENETEC; 2010.

13. Secretaría de Salud México. Guía de Práctica Clínica. Diagnóstico y tratamiento de síndrome de dificultad respiratoria en el recién nacido. Ciudad de México: CENETEC; 2009.

14. Bautista-Casasnovas AL. Estreñimiento. An. pediatr. contin. 2011; 9(4): 201-8.

15. Villoldo ME. Impacto del ruido ambiental en la Unidad de Cuidado Intensivo Neonatal. Enfermería Neonatal. 2011; 4(11): 27-30.

16. Reina NC. El proceso de enfermería: instrumento para el cuidado. Umbral científico. 2010; (17): 18-23.

17. Romero-Maldonado S, Arroyo-Cabrales LM, Reyna-Ríos ER. Consenso prematuro tardío. Perinatol. reprod. hum. 2010; 24(2): 124-130. 
18. Mata-Méndez M, Salazar-Barajas ME, Herrera-Pérez LR. Cuidado enfermero en el recién nacido prematuro. Rev. enferm. Inst. Mex. Seguro Soc. 2009; $17(1)$ : 45-54.

19. Gutiérrez-Muñoz F. Ventilación mecánica. Acta méd. peruana. 2011; 28(2): 87-104.

20. Vega-Angarita OM, González-Escobar DS. Teoría del Déficit de Autocuidado: interpretación desde los elementos conceptuales. Ciencia y Cuidado. 2007; 4(4): 28-35.

21. Fehlmann E, Tapia JL, Fernández R, et al. Impacto del síndrome de dificultad respiratoria en recién nacidos de muy bajo peso de nacimiento: estudio multicéntrico sudamericano. Arch. argent. pediatr. $2010 ; 108(5): 393-400$. 